\title{
Some Results for Nonlinear $(n+1)$-Term Fractional Integrodifferential Inclusions with Multipoint Boundary Conditions
}

\author{
Huacheng Zhou, Chunhai Kou, and Wen Song \\ Department of Applied Mathematics, Donghua University, Shanghai 201620, China \\ Correspondence should be addressed to Chunhai Kou, kouchunhai@hotmail.com
}

Received 18 April 2011; Accepted 24 May 2011

Academic Editors: B. Djafari-Rouhani, G. L. Karakostas, and W. Kryszewski

Copyright (c) 2011 Huacheng Zhou et al. This is an open access article distributed under the Creative Commons Attribution License, which permits unrestricted use, distribution, and reproduction in any medium, provided the original work is properly cited.

We are concerned with the nonlinear $(n+1)$-term fractional integrodifferential inclusions $L(D) u \in$ $F(t, u, T u, S u)$, a.e. $t \in[0,1]$, where $L(D)=D^{\alpha}-b_{n} D^{\beta_{n}}-b_{n-1} D^{\beta_{n-1}}-\cdots-b_{1} D^{\beta_{1}}, 0<\beta_{1}<\beta_{2}<\cdots<$ $\beta_{n-1}<\beta_{n}<1<\alpha<2, b_{1}, b_{2}, \ldots, b_{n}$ are constant coefficients, and $(T u)(t)=\int_{0}^{1} k(t, s) u(s) d s,(S u)(t)=$ $\int_{0}^{t} l(t, s) u(s) d s$, subject to the nonlocal conditions $u(0)=0, u(1)=\sum_{i=1}^{m} \gamma_{i} u\left(\eta_{i}\right)$. The existence results are obtained by using two fixed-point theorems due to Bohnenblust-Karlin and CovitzNadler, respectively. Our results partly generalize and improve the known ones.

\section{Introduction}

Fractional differential equations (FDEs) have received increasing interest for the last three decades. It is benefited by the intensive development of the theory of fractional calculus itself and by the applications of such constructions in the modeling of many phenomena in various fields of science, engineering economics, and other fields, see for instance [1-12] and references therein.

Babakhani and Gejji [7] considered the existence of positive solutions for the nonlinear fractional differential equations

$$
L(D) u=f(t, u), \quad u(0)=0,0<t<1
$$

where $L(D)=D^{s_{n}}-a_{n-1} D^{s_{n-1}}-\cdots-a_{1} D^{s_{1}}, 0<s_{1}<s_{2}<\cdots<s_{n}<1, a_{j}>0, j=1,2, \ldots, n-1$, and $D^{s_{j}}$ is the standard Riemann-Liouville fractional derivative. Some existence results of positive solutions are obtained by using some fixed-point theorems on a cone. 
Stojanović [11] considered the existenceuniqueness of solutions for a nonlinear $n$-term fractional differential equation

$$
b_{0} D^{\beta_{0}} u(t)+\sum_{i=1}^{m-1} b_{i} D^{\beta_{i}} u(t)+\sum_{i=m}^{n-1} b_{i} D^{\alpha_{i}} u(t)+b_{n} D^{\alpha_{n}} u(t)=f(t, u(t)), \quad t \in(0,1),
$$

where $0<\beta_{1}<\beta_{2}<\cdots<\beta_{m-1}<1<\alpha_{m}<\alpha_{m+1}<\cdots<\alpha_{n}<2$, with initial data $u(0)=f(0)$, $u_{t}(0)=g(0)$.

On the other hand, realistic problems arising from economics, optimal control, and so on can be modeled as differential inclusions. Recently, El-Sayed and Ibrahim [13] initiated the study of fractional differential equations inclusions. Differential inclusions have been widely investigated by many authors, see [14-30] and references therein.

Very recently, in the survey paper [16], Agarwal et al. establish sufficient conditions for the existence and uniqueness of solutions for various classes of initial and boundary value problems for fractional differential equations and inclusions involving the Caputo fractional derivative.

Ouahab [26] studied the following boundary value problem of fractional differential inclusions:

$$
\begin{gathered}
-D^{\alpha} y(t) \in F(t, y(t)), \quad \text { a.e. } t \in J=[0,1], 1<\alpha \leq 2, \\
y(0)=y(1)=0,
\end{gathered}
$$

where $D^{\alpha}$ is the standard Riemann-Liouville fractional derivative, $F: J \times \mathbb{R} \rightarrow p(\mathbb{R})$ is a multivalued map with compact values.

Chang and Nieto [27] studied boundary value problem of fractional differential inclusions

$$
\begin{gathered}
{ }_{0}^{C} D^{\delta} y(t) \in F(t, y(t)), \quad t \in J:=[0,1], \delta \in(1,2), \\
y(0)=\alpha, \quad y(1)=\beta, \quad \alpha, \beta \neq 0,
\end{gathered}
$$

where ${ }_{0}^{C} D^{\delta} y(t)$ is the Caputo's derivative, $F: J \times \mathbb{R} \rightarrow p(\mathbb{R})$.

However, to the best of our knowledge, the existence of solutions for fractional integro-differential inclusions with multipoint boundary conditions has not been paid much attention. Our goal is to fill this gap in literature.

In the present work, we consider more general fractional integro-differential inclusions

$$
L(D) u \in F(t, u, T u, S u), \quad \text { a.e. } t \in[0,1]
$$

with multipoint boundary conditions

$$
u(0)=0, \quad u(1)=\sum_{i=1}^{m} \gamma_{i} u\left(\eta_{i}\right)
$$

where $L(D)=D^{\alpha}-b_{n} D^{\beta_{n}}-b_{n-1} D^{\beta_{n-1}}-\cdots-b_{1} D^{\beta_{1}}, 0<\beta_{1}<\beta_{2}<\cdots<\beta_{n-1}<\beta_{n}<1<\alpha<2$, $D^{\alpha}, D^{\beta_{j}}, j=1,2, \ldots, n$, is the standard Riemann-Liouville fractional derivative, $0<\eta_{1}<$ $\eta_{2} \cdots<\eta_{m}<1,0 \leq \sum_{i=1}^{m} \gamma_{i} \eta_{i}^{\alpha-1}<1,(T u)(t)=\int_{0}^{1} k(t, s) u(s) \mathrm{d} s,(S u)(t)=\int_{0}^{t} l(t, s) u(s) \mathrm{d} s$, 
$k, l$ are two continuous functions on $[0,1] \times[0,1]$, and $F:[0,1] \times \mathbb{R} \times \mathbb{R} \times \mathbb{R} \rightarrow D(\mathbb{R})$ is a given multivalued function $(\mathcal{P}(\mathbb{R})$ is the family of all nonempty subsets of $\mathbb{R})$. We shall consider both the cases of convex and nonconvex valued right hand side and establish some sufficient conditions which admit that the integro-differential inclusions problem has at least one solution. These results obtained by applying two fixed point theorems due to Bohnenblust-Karlin and Covitz-Nadler are complement of previously known results.

The organization of this paper is as follows. In Section 2, we present some necessary definitions and preliminaries which are used throughout this paper. Main results and their proofs are given in Section 3.

\section{Preliminaries and Several Lemmas}

In this section, we recall some basic definitions and notations and give several lemmas which are useful in our discussion.

Definition 2.1. The Riemann-Liouville fractional integral of order $\alpha>0$ of a function $f: \mathbb{R}^{+} \rightarrow$ $\mathbb{R}$ is given by

$$
I^{\alpha} f(t)=\frac{1}{\Gamma(\alpha)} \int_{0}^{t}(t-s)^{\alpha-1} f(s) \mathrm{d} t
$$

provided the right side is pointwise defined on $\mathbb{R}^{+}$.

Definition 2.2. The Riemann-Liouville fractional derivative of function $f: \mathbb{R}^{+} \rightarrow \mathbb{R}$ is given by

$$
D^{\alpha} f(t)=D^{n} I^{n-\alpha} f(t),
$$

where $n=[\alpha]+1, D^{n}=\mathrm{d}^{n} / \mathrm{d} t^{n}$, provided the right side is pointwise defined on $\mathbb{R}^{+}$.

Lemma 2.3. Let $\alpha>0$, then

$$
I^{\alpha} D^{\alpha} x(t)=x(t)+c_{1} t^{\alpha-1}+c_{2} t^{\alpha-2}+\cdots+c_{n} t^{\alpha-n}
$$

for some $c_{i} \in \mathbb{R}, i=0,1,2, \ldots, n-1, n=-[-\alpha]$.

Remark 2.4. If the fractional derivative $D^{\alpha} x(t)$ is integrable, then

$$
I^{\alpha} D^{\beta} x(t)=I^{\alpha-\beta} x(t)-\left[I^{1-\beta} x(t)\right]_{t=0} \frac{t^{\alpha-1}}{\Gamma(\alpha)}, \quad 0<\beta \leq \alpha<1 .
$$

If $x$ is continuous on $[0,1]$, then $\left[I^{1-\beta} x(t)\right]_{t=0}=0$, and (2.4) reduces to

$$
I^{\alpha} D^{\beta} x(t)=I^{\alpha-\beta} x(t), \quad 0<\beta \leq \alpha<1 .
$$


The reader is referred to $[4,8,9]$ for more details on fractional integrals and fractional derivatives.

Lemma 2.5. Let $g \in L([0,1], \mathbb{R}), 0<\beta_{1}<\beta_{2}<\cdots<\beta_{n-1}<\beta_{n}<1<\alpha<2$, and $0<\sum_{i=1}^{m} \gamma_{i} \eta_{i}^{\alpha-1}<$ 1. Then $u(t)$ is a solution of the $B V P$

$$
\begin{gathered}
\left(D^{\alpha}-b_{n} D^{\beta_{n}}-b_{n-1} D^{\beta_{n-1}}-\cdots-b_{1} D^{\beta_{1}}\right) u(t)=g(t), \quad \text { a.e. } t \in[0,1] \\
u(0)=0, \quad u(1)=\sum_{i=1}^{m} \gamma_{i} u\left(\eta_{i}\right)
\end{gathered}
$$

if and only if $u(t)$ satisfies the integral equation

$$
\begin{aligned}
u(t)= & \sum_{i=1}^{m} \sum_{j=1}^{n} \frac{\gamma_{i} b_{j}}{\left(1-\sum_{i=1}^{m} \gamma_{i} \eta_{i}^{\alpha-1}\right) \Gamma\left(\alpha-\beta_{j}\right)} \int_{0}^{\eta_{i}}\left(\eta_{i}-s\right)^{\alpha-\beta_{j}-1} t^{\alpha-1} u(s) \mathrm{d} s \\
& -\sum_{i=1}^{n} \frac{b_{i}}{\left(1-\sum_{i=1}^{m} \gamma_{i} \eta_{i}^{\alpha-1}\right) \Gamma\left(\alpha-\beta_{i}\right)} \int_{0}^{1}(1-s)^{\alpha-\beta_{i}-1} t^{\alpha-1} u(s) \mathrm{d} s \\
& +\sum_{i=1}^{n} \frac{b_{i}}{\Gamma\left(\alpha-\beta_{i}\right)} \int_{0}^{t}(t-s)^{\alpha-\beta_{i}-1} u(s) \mathrm{d} s+\frac{1}{\Gamma(\alpha)} \int_{0}^{t}(t-s)^{\alpha-1} g(s) \mathrm{d} s \\
& +\sum_{i=1}^{m} \frac{\gamma_{i}}{\left(1-\sum_{i=1}^{m} \gamma_{i} \eta_{i}^{\alpha-1}\right) \Gamma(\alpha)} \int_{0}^{\eta_{i}}\left(\eta_{i}-s\right)^{\alpha-1} t^{\alpha-1} g(s) \mathrm{d} s \\
& -\frac{1}{\left(1-\sum_{i=1}^{m} \gamma_{i} \eta_{i}^{\alpha-1}\right) \Gamma(\alpha)} \int_{0}^{1}(1-s)^{\alpha-1} t^{\alpha-1} g(s) \mathrm{d} s .
\end{aligned}
$$

Proof. In view of Lemma 2.3 and Remark 2.4, (2.6) is equivalent to the integral equation

$$
u(t)=c_{1} t^{\alpha-1}+c_{2} t^{\alpha-2}+\sum_{i=1}^{n} b_{i} I^{\alpha-\beta_{i}} u(t)+I^{\alpha} g(t)
$$

for some $c_{1}, c_{2} \in \mathbb{R}$, that is,

$$
u(t)=c_{1} t^{\alpha-1}+c_{2} t^{\alpha-2}+\sum_{i=1}^{n} \frac{b_{i}}{\Gamma\left(\alpha-\beta_{i}\right)} \int_{0}^{t}(t-s)^{\alpha-\beta_{i}-1} u(s) \mathrm{d} s+\frac{1}{\Gamma(\alpha)} \int_{0}^{t}(t-s)^{\alpha-1} g(s) \mathrm{d} s .
$$

The boundary condition $u(0)=0$ implies $c_{2}=0$. Thus,

$$
u(t)=c_{1} t^{\alpha-1}+\sum_{i=1}^{n} \frac{b_{i}}{\Gamma\left(\alpha-\beta_{i}\right)} \int_{0}^{t}(t-s)^{\alpha-\beta_{i}-1} u(s) \mathrm{d} s+\frac{1}{\Gamma(\alpha)} \int_{0}^{t}(t-s)^{\alpha-1} g(s) \mathrm{d} s .
$$


In view of the boundary condition $u(1)=\sum_{i=1}^{m} \gamma_{i} u\left(\eta_{i}\right)$, we conclude that

$$
\begin{aligned}
c_{1}= & \sum_{i=1}^{m} \sum_{j=1}^{n} \frac{\gamma_{i} b_{j}}{\left(1-\sum_{i=1}^{m} \gamma_{i} \eta_{i}^{\alpha-1}\right) \Gamma\left(\alpha-\beta_{j}\right)} \int_{0}^{\eta_{i}}\left(\eta_{i}-s\right)^{\alpha-\beta_{j}-1} u(s) \mathrm{d} s \\
& -\sum_{i=1}^{n} \frac{b_{i}}{\left(1-\sum_{i=1}^{m} \gamma_{i} \eta_{i}^{\alpha-1}\right) \Gamma\left(\alpha-\beta_{i}\right)} \int_{0}^{1}(1-s)^{\alpha-\beta_{i}-1} u(s) \mathrm{d} s \\
& +\sum_{i=1}^{m} \frac{\gamma_{i}}{\left(1-\sum_{i=1}^{m} \gamma_{i} \eta_{i}^{\alpha-1}\right) \Gamma(\alpha)} \int_{0}^{\eta_{i}}\left(\eta_{i}-s\right)^{\alpha-1} g(s) \mathrm{d} s \\
& -\frac{1}{\left(1-\sum_{i=1}^{m} \gamma_{i} \eta_{i}^{\alpha-1}\right) \Gamma(\alpha)} \int_{0}^{1}(1-s)^{\alpha-1} g(s) \mathrm{d} s .
\end{aligned}
$$

Therefore, the solution $u(t)$ of (2.6) and (2.7) satisfies (2.8).

Conversely, if $u(t)$ is a solution of (2.8), it is easy to verify that $u(t)$ satisfies (2.6) and (2.7). The proof is complete.

Now, we recall some facts from multivalued analysis.

Let $(X, d)$ be a metric space, and $D(X)=\{Y \subset X: Y \neq \emptyset\}, p_{b d}(X)=\{Y \in D(X)$ : $Y$ bounded $\}, D_{c l}(X)=\{Y \in D(X): Y$ closed $\}, D_{c v}(X)=\{Y \in P(X): Y$ convex $\}, p_{c p}(X)=$ $\{Y \in P(X): Y$ compact $\}, p_{c v, c p}(X)=p_{c v}(X) \cap p_{c p}(X)$, and so forth.

Consider $H_{d}: \mathcal{D}(X) \times \mathcal{D}(X) \rightarrow \mathbb{R}_{+} \cup\{\infty\}$, given by

$$
H_{d}(A, B)=\max \left\{\sup _{x \in A} d(x, B), \sup _{y \in B} d(A, y)\right\}
$$

where $d(x, B)=\inf _{y \in B} d(x, y), d(A, y)=\inf _{x \in A} d(x, y)$. Then $\left(p_{b d, c l}(X), H_{d}\right)$ is a metric space and $\left(P_{c l}(X), H_{d}\right)$ is a generalized metric space (see [31]).

A multivalued map $N:[0,1] \rightarrow D_{c l}(X)$ is said to be measurable if for each $u \in X$, the function $Y:[0,1] \rightarrow \mathbb{R}$, defined by

$$
Y(t)=d(u, N(t))=\inf \{d(u, z): z \in N(t)\}
$$

is measurable.

A multivalued map $F: X \rightarrow p(X)$ is convex (closed) valued if $F(u)$ is convex (closed) for all $u \in X$. $F$ is bounded on bounded sets if $F(B)=\cup_{u \in B} F(u)$ is bounded in $X$ for all $B \in P(X)$. That is, $\sup _{u \in B}\{\sup \{|y|: y \in F(u)\}\}<\infty$. F is called upper semicontinuous (u.s.c for short) on $X$ if for each $u_{0} \in X$, the set $F\left(u_{0}\right)$ is nonempty closed subset of $X$, and if for each open set $\mathcal{U}$ of $X$ containing $F\left(u_{0}\right)$, there exists an open neighborhood $\mho$ of $u_{0}$ such that $F(\mathcal{U}) \subset \mathcal{U}$. $G$ is said to be completely continuous if $F(B)$ is relatively compact for every $B \in p_{b d}(X)$. If the multivalued map $F$ is completely continuous with nonempty compact valued, then $G$ is u.s.c. if and only if $F$ has closed graph, that is, $x_{n} \rightarrow x_{*}, y_{n} \rightarrow y_{*}, y_{n} \in G\left(x_{*}\right)$ imply $y_{*} \in G\left(x_{*}\right)$.

More details on multivalued maps can be found in the books of Deimling [3], Górniewicz [32], Hu and Papageorgiou [33], and Tolstonogov [34]. 
Definition 2.6. The multivalued map $F:[0,1] \times \mathbb{R} \times \mathbb{R} \times \mathbb{R} \rightarrow \mathcal{D}(\mathbb{R})$ is $L^{1}$-Carathéodory if

(i) $t \mapsto F(t, u, v, w)$ is measurable for each $(u, v, w) \in \mathbb{R} \times \mathbb{R} \times \mathbb{R}$;

(ii) $(u, v, w) \mapsto F(t, u, v, w)$ is upper semicontinuous for almost all $t \in[0,1]$;

(iii) for each $r>0$, there exists $\varphi_{r} \in L^{1}\left([0,1], \mathbb{R}_{+}\right)$such that for all $|u|,|v|,|w| \leq r$ and for almost all $t \in[0,1]$,

$$
\|F(t, u, v, w)\|_{p}=\sup \{|f|: f \in F(t, u, v, w)\} \leq \varphi_{r}(t) .
$$

For any $u \in C([0,1], \mathbb{R})$, we define the set

$$
S_{F, u}=\left\{f \in L^{1}([0,1], \mathbb{R}): f(t) \in F(t, u(t),(T u)(t),(S u)(t)) \text { for a.e. } t \in[0,1]\right\}
$$

which is known as the set of selection functions.

Lemma 2.7 (see [35]). Let $X$ be a Banach space. Let $F:[0,1] \times X \rightarrow p_{c p, c v}(X)$ be an $L^{1}$-Carathéodory multivalued map with $S_{F, u} \neq \emptyset$, and let $\Gamma$ be a linear continuous mapping from $L^{1}([0,1], X)$ into $C([0,1], X)$. Then the operator

$$
\begin{aligned}
\Gamma \circ S_{F}: C([0,1], X) \longrightarrow p_{c p, c v}(C([0,1], X)), \\
u \longmapsto\left(\Gamma \circ S_{F}\right)(y):=\Gamma\left(S_{F, u}\right)
\end{aligned}
$$

is a closed graph operator in $C([0,1], X) \times C([0,1], X)$.

The following Bohnenblust-Karlin fixed-point lemma and the fixed-point theorem for contractive multivalued operators given by Covitz and Nadler are of great importance in the proofs of our main results. The proofs of these results can be found in Bohnenblust and Karlin [30] and in Covitz and Nadler [36].

Lemma 2.8 (see [30]). Let $X$ be a Banach space, $D$ be a nonempty subset of $X$, which is bounded, closed, and convex. Suppose $G: D \rightarrow D(X)$ is u.s.c. with closed, convex values, and such that $G(D) \subset D$ and $G(D)$ is relatively compact. Then $G$ has a fixed point.

Lemma 2.9 (see [36]). Let $(X, d)$ be a complete metric space. If $N$ is a contraction operator, then Fix $N \neq \emptyset$.

For convenience, let us list some conditions.

(H1) $\sum_{i=1}^{m} \sum_{j=1}^{n}\left(\gamma_{i} b_{j} \eta_{i}^{\alpha-\beta_{j}} /\left(1-\sum_{i=1}^{m} \gamma_{i} \eta_{i}^{\alpha-1}\right) \Gamma\left(\alpha-\beta_{j}+1\right)\right)+\sum_{i=1}^{n}\left(b_{i} /\left(1-\sum_{i=1}^{m} \gamma_{i} \eta_{i}^{\alpha-1}\right) \Gamma\left(\alpha-\beta_{i}+\right.\right.$ $1))+\sum_{i=1}^{n}\left(b_{i} / \Gamma\left(\alpha-\beta_{i}+1\right)\right)<1$;

(H2) $F:[0,1] \times \mathbb{R} \times \mathbb{R} \times \mathbb{R} \rightarrow p_{c p, c v}(\mathbb{R})$ is Carethéodory multivalued map. 
Lemma 2.10. Assume that hypothesis (H1) is satisfied. For any $g \in C([0,1], \mathbb{R})$, the integral equation

$$
\begin{aligned}
u(t)= & \sum_{i=1}^{m} \sum_{j=1}^{n} \frac{\gamma_{i} b_{j}}{\left(1-\sum_{i=1}^{m} \gamma_{i} \eta_{i}^{\alpha-1}\right) \Gamma\left(\alpha-\beta_{j}\right)} \int_{0}^{\eta_{i}}\left(\eta_{i}-s\right)^{\alpha-\beta_{j}-1} t^{\alpha-1} u(s) \mathrm{d} s \\
& -\sum_{i=1}^{n} \frac{b_{i}}{\left(1-\sum_{i=1}^{m} \gamma_{i} \eta_{i}^{\alpha-1}\right) \Gamma\left(\alpha-\beta_{i}\right)} \int_{0}^{1}(1-s)^{\alpha-\beta_{i}-1} t^{\alpha-1} u(s) \mathrm{d} s \\
& +\sum_{i=1}^{n} \frac{b_{i}}{\Gamma\left(\alpha-\beta_{i}\right)} \int_{0}^{t}(t-s)^{\alpha-\beta_{i}-1} u(s) \mathrm{d} s+g(t)
\end{aligned}
$$

has a unique solution in $C([0,1], \mathbb{R})$.

Proof. We define the operator $A$ as follows:

$$
\begin{aligned}
(A u)(t):= & \sum_{i=1}^{m} \sum_{j=1}^{n} \frac{\gamma_{i} b_{j}}{\left(1-\sum_{i=1}^{m} \gamma_{i} \eta_{i}^{\alpha-1}\right) \Gamma\left(\alpha-\beta_{j}\right)} \int_{0}^{\eta_{i}}\left(\eta_{i}-s\right)^{\alpha-\beta_{j}-1} t^{\alpha-1} u(s) \mathrm{d} s \\
& -\sum_{i=1}^{n} \frac{b_{i}}{\left(1-\sum_{i=1}^{m} \gamma_{i} \eta_{i}^{\alpha-1}\right) \Gamma\left(\alpha-\beta_{i}\right)} \int_{0}^{1}(1-s)^{\alpha-\beta_{i}-1} t^{\alpha-1} u(s) \mathrm{d} s \\
& +\sum_{i=1}^{n} \frac{b_{i}}{\Gamma\left(\alpha-\beta_{i}\right)} \int_{0}^{t}(t-s)^{\alpha-\beta_{i}-1} u(s) \mathrm{d} s+g(t)
\end{aligned}
$$

Obviously, $A$ is a map from $C([0,1], \mathbb{R})$ into $C([0,1], \mathbb{R})$. Also, we have

$$
\begin{aligned}
\left|\left(A u_{1}\right)(t)-\left(A u_{2}\right)(t)\right| \leq & \sum_{i=1}^{m} \sum_{j=1}^{n} \frac{\gamma_{i} b_{j}}{\left(1-\sum_{i=1}^{m} \gamma_{i} \eta_{i}^{\alpha-1}\right) \Gamma\left(\alpha-\beta_{j}\right)} \int_{0}^{\eta_{i}}\left(\eta_{i}-s\right)^{\alpha-\beta_{j}-1} t^{\alpha-1}\left|u_{1}(s)-u_{2}(s)\right| \mathrm{d} s \\
& +\sum_{i=1}^{n} \frac{b_{i}}{\left(1-\sum_{i=1}^{m} \gamma_{i} \eta_{i}^{\alpha-1}\right) \Gamma\left(\alpha-\beta_{i}\right)} \int_{0}^{1}(1-s)^{\alpha-\beta_{i}-1} t^{\alpha-1}\left|u_{1}(s)-u_{2}(s)\right| \mathrm{d} s \\
& +\sum_{i=1}^{n} \frac{b_{i}}{\Gamma\left(\alpha-\beta_{i}\right)} \int_{0}^{t}(t-s)^{\alpha-\beta_{i}-1}\left|u_{1}(s)-u_{2}(s)\right| \mathrm{d} s \\
\leq & \left(\sum_{i=1}^{m} \sum_{j=1}^{n} \frac{\gamma_{i} b_{j} \eta_{i}^{\alpha-\beta_{j}}}{\left(1-\sum_{i=1}^{m} \gamma_{i} \eta_{i}^{\alpha-1}\right) \Gamma\left(\alpha-\beta_{j}+1\right)}\right. \\
& \left.+\sum_{i=1}^{n} \frac{b_{i}}{\left(1-\sum_{i=1}^{m} \gamma_{i} \eta_{i}^{\alpha-1}\right) \Gamma\left(\alpha-\beta_{i}+1\right)}+\sum_{i=1}^{n} \frac{b_{i}}{\Gamma\left(\alpha-\beta_{i}+1\right)}\right)\left\|u_{1}-u_{2}\right\| .
\end{aligned}
$$


Therefore, $\left\|A u_{1}-A u_{2}\right\| \leq \lambda\left\|u_{1}-u_{2}\right\|$, where $\lambda=\sum_{i=1}^{m} \sum_{j=1}^{n}\left(\gamma_{i} b_{j} \eta_{i}^{\alpha-\beta_{j}} /\left(1-\sum_{i=1}^{m} \gamma_{i} \eta_{i}^{\alpha-1}\right) \Gamma(\alpha-\right.$ $\left.\left.\beta_{j}+1\right)\right)+\sum_{i=1}^{n}\left(b_{i} /\left(1-\sum_{i=1}^{m} \gamma_{i} \eta_{i}^{\alpha-1}\right) \Gamma\left(\alpha-\beta_{i}+1\right)\right)+\sum_{i=1}^{n}\left(b_{i} / \Gamma\left(\alpha-\beta_{i}+1\right)\right)$. By (H1) and Banach contraction principle, the conclusion of lemma is true.

Definition 2.11. A function $u \in C[0,1]$ is said to be a solution of the BVP (1.5) and (1.6), if there exists a function $f \in L^{1}([0,1], \mathbb{R})$ such that $f(t) \in F(t, u(t),(T u)(t),(S u)(t))$ a.e. on $[0,1]$ and

$$
\begin{aligned}
u(t)= & \sum_{i=1}^{m} \sum_{j=1}^{n} \frac{\gamma_{i} b_{j}}{\left(1-\sum_{i=1}^{m} \gamma_{i} \eta_{i}^{\alpha-1}\right) \Gamma\left(\alpha-\beta_{j}\right)} \int_{0}^{\eta_{i}}\left(\eta_{i}-s\right)^{\alpha-\beta_{j}-1} t^{\alpha-1} u(s) \mathrm{d} s \\
& -\sum_{i=1}^{n} \frac{b_{i}}{\left(1-\sum_{i=1}^{m} \gamma_{i} \eta_{i}^{\alpha-1}\right) \Gamma\left(\alpha-\beta_{i}\right)} \int_{0}^{1}(1-s)^{\alpha-\beta_{i}-1} t^{\alpha-1} u(s) \mathrm{d} s \\
& +\sum_{i=1}^{n} \frac{b_{i}}{\Gamma\left(\alpha-\beta_{i}\right)} \int_{0}^{t}(t-s)^{\alpha-\beta_{i}-1} u(s) \mathrm{d} s+\frac{1}{\Gamma(\alpha)} \int_{0}^{t}(t-s)^{\alpha-1} f(s) \mathrm{d} s \\
& +\sum_{i=1}^{m} \frac{\gamma_{i}}{\left(1-\sum_{i=1}^{m} \gamma_{i} \eta_{i}^{\alpha-1}\right) \Gamma(\alpha)} \int_{0}^{\eta_{i}}\left(\eta_{i}-s\right)^{\alpha-1} t^{\alpha-1} f(s) \mathrm{d} s \\
& -\frac{1}{\left(1-\sum_{i=1}^{m} \gamma_{i} \eta_{i}^{\alpha-1}\right) \Gamma(\alpha)} \int_{0}^{1}(1-s)^{\alpha-1} t^{\alpha-1} f(s) \mathrm{d} s .
\end{aligned}
$$

\section{Main Results}

In this section, we present our main results and prove them. Firstly, under convexity condition on the multivalued right-hand side, we are to establish the existence theorem of solutions for fractional differential inclusions (1.5) and (1.6), by employing the Bohnenblust-Karlin fixed-point theorem. Then, under nonconvexity condition on the multivalued right-hand side, the existence theorem of solutions are gotten, by employing the Covitz-Nadler fixedpoint theorem.

Theorem 3.1. Assume that hypotheses (H1) and (H2) are satisfied. Then BVP (1.5) and (1.6) has at least one solution in $C([0,1], \mathbb{R})$, provided that

(H3) $\tau / \Gamma(\alpha)+\sum_{i=1}^{m}\left(\gamma_{i} \mu_{i} /\left(1-\sum_{i=1}^{m} \gamma_{i} \eta_{i}^{\alpha-1}\right) \Gamma(\alpha)\right)+\left(\omega /\left(1-\sum_{i=1}^{m} \gamma_{i} \eta_{i}^{\alpha-1}\right) \Gamma(\alpha)\right)<1-\lambda$, where

$$
\begin{gathered}
\tau=\lim _{r \rightarrow \infty} \inf \int_{0}^{1} \frac{\max \left\{\varphi_{r}(s), \varphi_{\tilde{k} r}(s), \varphi_{\tilde{l} r}(s)\right\}}{r} \mathrm{~d} s, \\
\mu_{i}=\lim _{r \rightarrow \infty} \inf \int_{0}^{\eta_{i}} \frac{\left(\eta_{i}-s\right)^{\alpha-1} \max \left\{\varphi_{r}(s), \varphi_{\tilde{k} r}(s), \varphi_{\tilde{l} r}(s)\right\}}{r} \mathrm{~d} s, \\
\omega=\lim _{r \rightarrow \infty} \inf \int_{0}^{1} \frac{(1-s)^{\alpha-1} \max \left\{\varphi_{r}(s), \varphi_{\tilde{k} r}(s), \varphi_{\tilde{l} r}(s)\right\}}{r} \mathrm{~d} s, \\
\tilde{k}=\sup _{t \in[0,1]} \int_{0}^{1} k(t, s) \mathrm{d} s, \quad \tilde{l}=\sup _{t \in[0,1]} \int_{0}^{t} l(t, s) \mathrm{d} s,
\end{gathered}
$$


and $\lambda$ is defined in the proof of Lemma 2.10.

Proof. To transform the problem into a fixed-point problem, we consider the multivalued operator, $N: C([0,1], \mathbb{R}) \rightarrow D(C([0,1], \mathbb{R}))$, where for any $u \in C([0,1], \mathbb{R}), N(u)$ is defined by

$$
\begin{aligned}
\{h \in C([0,1], \mathbb{R}): h(t)= & \sum_{i=1}^{m} \sum_{j=1}^{n} \frac{\gamma_{i} b_{j}}{\left(1-\sum_{i=1}^{m} \gamma_{i} \eta_{i}^{\alpha-1}\right) \Gamma\left(\alpha-\beta_{j}\right)} \int_{0}^{\eta_{i}}\left(\eta_{i}-s\right)^{\alpha-\beta_{j}-1} t^{\alpha-1} h(s) \mathrm{d} s \\
& -\sum_{i=1}^{n} \frac{b_{i}}{\left(1-\sum_{i=1}^{m} \gamma_{i} \eta_{i}^{\alpha-1}\right) \Gamma\left(\alpha-\beta_{i}\right)} \int_{0}^{1}(1-s)^{\alpha-\beta_{i}-1} t^{\alpha-1} h(s) \mathrm{d} s \\
& +\sum_{i=1}^{n} \frac{b_{i}}{\Gamma\left(\alpha-\beta_{i}\right)} \int_{0}^{t}(t-s)^{\alpha-\beta_{i}-1} h(s) \mathrm{d} s+\frac{1}{\Gamma(\alpha)} \int_{0}^{t}(t-s)^{\alpha-1} f(s) \mathrm{d} s \\
& +\sum_{i=1}^{m} \frac{\gamma_{i}}{\left(1-\sum_{i=1}^{m} \gamma_{i} \eta_{i}^{\alpha-1}\right) \Gamma(\alpha)} \int_{0}^{\eta_{i}}\left(\eta_{i}-s\right)^{\alpha-1} t^{\alpha-1} f(s) \mathrm{d} s \\
& \left.-\frac{1}{\left(1-\sum_{i=1}^{m} \gamma_{i} \eta_{i}^{\alpha-1}\right) \Gamma(\alpha)} \int_{0}^{1}(1-s)^{\alpha-1} t^{\alpha-1} f(s) \mathrm{d} s, f \in S_{F, u}\right\} .
\end{aligned}
$$

For any $f \in L^{1}([0,1], \mathbb{R})$, we have $\int_{0}^{t}(t-s)^{\alpha-1} f(s) \mathrm{d} s, \int_{0}^{\eta_{i}}\left(\eta_{i}-s\right)^{\alpha-1} t^{\alpha-1} f(s) \mathrm{d} s, \int_{0}^{1}(1-$ $s)^{\alpha-1} t^{\alpha-1} f(s) \mathrm{d} s \in C([0,1], \mathbb{R})$. In view of Lemma 2.10, $N$ is well defined. Moreover, it follows from the convexity of $S_{F, u}$ (because $F$ has convex values) that $N(u)$ is convex for each $u \in$ $C([0,1], \mathbb{R})$. Clearly, the fixed points of $N$ are solutions of (1.5) and (1.6).

We shall show that $N$ has a fixed point in three steps.

Step 1. we claim that there exists a $r^{*}>0$, such that $N\left(B_{r^{*}}\right) \subset B_{r^{*}}$, where $B_{r^{*}}=\{u \in$ $\left.C([0,1], \mathbb{R}):\|u\| \leq r^{*}\right\}$.

In fact, if it is not true, then for any $r>0$, there exists a function $u_{r} \in B_{r}, h_{r} \in N\left(u_{r}\right)$ but $h_{r} \notin B_{r}$, that is $\left\|h_{r}\right\|>r$ and for some $f_{r} \in S_{F, u}$

$$
\begin{aligned}
h_{r}(t)= & \sum_{i=1}^{m} \sum_{j=1}^{n} \frac{\gamma_{i} b_{j}}{\left(1-\sum_{i=1}^{m} \gamma_{i} \eta_{i}^{\alpha-1}\right) \Gamma\left(\alpha-\beta_{j}\right)} \int_{0}^{\eta_{i}}\left(\eta_{i}-s\right)^{\alpha-\beta_{j}-1} t^{\alpha-1} h_{r}(s) \mathrm{d} s \\
& -\sum_{i=1}^{n} \frac{b_{i}}{\left(1-\sum_{i=1}^{m} \gamma_{i} \eta_{i}^{\alpha-1}\right) \Gamma\left(\alpha-\beta_{i}\right)} \int_{0}^{1}(1-s)^{\alpha-\beta_{i}-1} t^{\alpha-1} h_{r}(s) \mathrm{d} s \\
& +\sum_{i=1}^{n} \frac{b_{i}}{\Gamma\left(\alpha-\beta_{i}\right)} \int_{0}^{t}(t-s)^{\alpha-\beta_{i}-1} h_{r}(\mathrm{~s}) \mathrm{d} s+\frac{1}{\Gamma(\alpha)} \int_{0}^{t}(t-s)^{\alpha-1} f_{r}(s) \mathrm{d} s \\
& +\sum_{i=1}^{m} \frac{\gamma_{i}}{\left(1-\sum_{i=1}^{m} \gamma_{i} \eta_{i}^{\alpha-1}\right) \Gamma(\alpha)} \int_{0}^{\eta_{i}}\left(\eta_{i}-s\right)^{\alpha-1} t^{\alpha-1} f_{r}(s) \mathrm{d} s \\
& -\frac{1}{\left(1-\sum_{i=1}^{m} \gamma_{i} \eta_{i}^{\alpha-1}\right) \Gamma(\alpha)} \int_{0}^{1}(1-s)^{\alpha-1} t^{\alpha-1} f_{r}(s) \mathrm{d} s .
\end{aligned}
$$


On the other hand, from $(\mathrm{H} 2)$, we obtain

$$
\begin{aligned}
\left|h_{r}(t)\right| \leq & \lambda\left\|h_{r}\right\|+\frac{1}{\Gamma(\alpha)} \int_{0}^{t}(t-s)^{\alpha-1} f_{r}(s) \mathrm{d} s \\
& +\frac{1}{\left(1-\sum_{i=1}^{m} \gamma_{i} \eta_{i}^{\alpha-1}\right) \Gamma(\alpha)} \int_{0}^{1}(1-s)^{\alpha-1} t^{\alpha-1} f_{r}(s) \mathrm{d} s \\
& +\sum_{i=1}^{m} \frac{\gamma_{i}}{\left(1-\sum_{i=1}^{m} \gamma_{i} \eta_{i}^{\alpha-1}\right) \Gamma(\alpha)} \int_{0}^{\eta_{i}}\left(\eta_{i}-s\right)^{\alpha-1} t^{\alpha-1} f_{r}(s) \mathrm{d} s \\
\leq & \lambda\left\|h_{r}\right\|+\frac{1}{\Gamma(\alpha)} \int_{0}^{1}(|t-s|)^{\alpha-1} \varphi_{\max \{r, \tilde{k} r, \tilde{l} r\}}(s) \mathrm{d} s \\
& +\sum_{i=1}^{m} \frac{\gamma_{i}}{\left(1-\sum_{i=1}^{m} \gamma_{i} \eta_{i}^{\alpha-1}\right) \Gamma(\alpha)} \int_{0}^{\eta_{i}}\left(\eta_{i}-s\right)^{\alpha-1} t^{\alpha-1} \varphi_{\max \{r, \tilde{k} r, \tilde{l} r\}}(s) \mathrm{d} s \\
& +\frac{1}{\left(1-\sum_{i=1}^{m} \gamma_{i} \eta_{i}^{\alpha-1}\right) \Gamma(\alpha)} \int_{0}^{1}(1-s)^{\alpha-1} t^{\alpha-1} \varphi_{\max \{r, \tilde{k} r, \tilde{r}\}}(s) \mathrm{d} s .
\end{aligned}
$$

Thus,

$$
\begin{aligned}
r<\left\|h_{r}\right\| \leq & \frac{1}{(1-\lambda) \Gamma(\alpha)} \int_{0}^{1}(|t-s|)^{\alpha-1} \varphi_{\max \{r, \tilde{k} r, \tilde{l} r\}}(s) \mathrm{d} s \\
& +\sum_{i=1}^{m} \frac{\gamma_{i}}{(1-\lambda)\left(1-\sum_{i=1}^{m} \gamma_{i} \eta_{i}^{\alpha-1}\right) \Gamma(\alpha)} \int_{0}^{\eta_{i}}\left(\eta_{i}-s\right)^{\alpha-1} \varphi_{\max \{r, \tilde{k} r, \tilde{l} r\}}(s) \mathrm{d} s \\
& +\frac{1}{(1-\lambda)\left(1-\sum_{i=1}^{m} \gamma_{i} \eta_{i}^{\alpha-1}\right) \Gamma(\alpha)} \int_{0}^{1}(1-s)^{\alpha-1} \varphi_{\max \{r, \tilde{k} r, \tilde{l} r\}}(s) \mathrm{d} s .
\end{aligned}
$$

Dividing both sides by $r$ and taking the lower limit as $r \rightarrow+\infty$, we get

$$
1 \leq \frac{\tau}{(1-\lambda) \Gamma(\alpha)}+\sum_{i=1}^{m} \frac{\gamma_{i} \mu_{i}}{(1-\lambda)\left(1-\sum_{i=1}^{m} \gamma_{i} \eta_{i}^{\alpha-1}\right) \Gamma(\alpha)}+\frac{\omega}{(1-\lambda)\left(1-\sum_{i=1}^{m} \gamma_{i} \eta_{i}^{\alpha-1}\right) \Gamma(\alpha)}
$$

This is a contraction to (H3). Hence there exists a $r^{*}>0$ such that $N\left(B_{r^{*}}\right) \subset B_{r^{*}}$. 
Step 2. $N\left(B_{r^{*}}\right)$ is equicontinuous.

Let $t_{1}, t_{2} \in[0,1], t_{1}<t_{2}, u \in B_{r^{*}}$, and $h \in N(u)$. Then there exists $f \in S_{F, u}$ such that for each $t \in[0,1]$, we have

$$
\begin{aligned}
h(t)= & \sum_{i=1}^{m} \sum_{j=1}^{n} \frac{\gamma_{i} b_{j}}{\left(1-\sum_{i=1}^{m} \gamma_{i} \eta_{i}^{\alpha-1}\right) \Gamma\left(\alpha-\beta_{j}\right)} \int_{0}^{\eta_{i}}\left(\eta_{i}-s\right)^{\alpha-\beta_{j}-1} t^{\alpha-1} h(s) \mathrm{d} s \\
& -\sum_{i=1}^{n} \frac{b_{i}}{\left(1-\sum_{i=1}^{m} \gamma_{i} \eta_{i}^{\alpha-1}\right) \Gamma\left(\alpha-\beta_{i}\right)} \int_{0}^{1}(1-s)^{\alpha-\beta_{i}-1} t^{\alpha-1} h(s) \mathrm{d} s \\
& +\sum_{i=1}^{n} \frac{b_{i}}{\Gamma\left(\alpha-\beta_{i}\right)} \int_{0}^{t}(t-s)^{\alpha-\beta_{i}-1} h(s) \mathrm{d} s+\frac{1}{\Gamma(\alpha)} \int_{0}^{t}(t-s)^{\alpha-1} f(s) \mathrm{d} s \\
& +\sum_{i=1}^{m} \frac{\gamma_{i}}{\left(1-\sum_{i=1}^{m} \gamma_{i} \eta_{i}^{\alpha-1}\right) \Gamma(\alpha)} \int_{0}^{\eta_{i}}\left(\eta_{i}-s\right)^{\alpha-1} t^{\alpha-1} f(s) \mathrm{d} s \\
& -\frac{1}{\left(1-\sum_{i=1}^{m} \gamma_{i} \eta_{i}^{\alpha-1}\right) \Gamma(\alpha)} \int_{0}^{1}(1-s)^{\alpha-1} t^{\alpha-1} f(s) \mathrm{d} s .
\end{aligned}
$$

Therefore,

$$
\begin{aligned}
\left|h\left(t_{1}\right)-h\left(t_{2}\right)\right| \leq & a\left(t_{2}^{\alpha-1}-t_{1}^{\alpha-1}\right)+\frac{1}{\Gamma(\alpha)} \int_{t_{1}}^{t_{2}} \varphi_{\max \left\{r^{*}, \tilde{k} r^{*}, \tilde{r} r^{*}\right\}}(s) \mathrm{d} s \\
& +\frac{1}{\Gamma(\alpha)} \int_{0}^{t_{1}}\left(\left(t_{2}-s\right)^{\alpha-1}-\left(t_{1}-s\right)^{\alpha-1}\right) \varphi_{\max \left\{r^{*}, \tilde{r} r^{*}, \tilde{r} \tilde{r}^{*}\right\}}(s) \mathrm{d} s \\
& +\sum_{i=1}^{n} \frac{b_{i} r^{*}}{\Gamma\left(\alpha-\beta_{i}+1\right)}\left(t_{2}^{\alpha-\beta_{i}}-t_{1}^{\alpha-\beta_{i}}+2\left(t_{2}-t_{1}\right)^{\alpha-\beta_{i}}\right),
\end{aligned}
$$

where

$$
\begin{aligned}
a= & \sum_{i=1}^{m} \sum_{j=1}^{n} \frac{\gamma_{i} b_{j} \eta_{i}^{\alpha-\beta_{j}} r^{*}}{\left(1-\sum_{i=1}^{m} \gamma_{i} \eta_{i}^{\alpha-1}\right) \Gamma\left(\alpha-\beta_{j}+1\right)}+\sum_{i=1}^{n} \frac{b_{i} r^{*}}{\left(1-\sum_{i=1}^{m} \gamma_{i} \eta_{i}^{\alpha-1}\right) \Gamma\left(\alpha-\beta_{i}+1\right)} \\
& +\sum_{i=1}^{m} \frac{\gamma_{i}}{\left(1-\sum_{i=1}^{m} \gamma_{i} \eta_{i}^{\alpha-1}\right) \Gamma(\alpha)} \int_{0}^{\eta_{i}}\left(\eta_{i}-s\right)^{\alpha-1} \varphi_{\max \left\{r^{*}, \tilde{k} r^{*}, \tilde{l} r^{*}\right\}}(s) \mathrm{d} s \\
& +\frac{1}{\left(1-\sum_{i=1}^{m} \gamma_{i} \eta_{i}^{\alpha-1}\right) \Gamma(\alpha)} \int_{0}^{1}(1-s)^{\alpha-1} \varphi_{\max \left\{r^{*}, \tilde{k} r^{*}, \tilde{l}, \tilde{l}^{*}\right\}}(s) \mathrm{d} s .
\end{aligned}
$$

The right-hand side of the above inequality tends to zeros independently of $h \in B_{r^{*}}$, as $t_{2}-t_{1} \rightarrow 0$. This shows that $N\left(B_{r^{*}}\right)$ is equicontinuous. 
Step 3. $N$ has a closed graph.

Let $u_{n} \rightarrow u_{*}, h_{n} \in N\left(u_{n}\right)$ and $h_{n} \rightarrow h_{*}$. We will prove that $h_{*} \in N\left(u_{*}\right)$. Now $h_{n} \in$ $N\left(u_{n}\right)$ implies that there exists $f_{n} \in S_{F, u_{n}}$ such that for each $t \in[0,1]$,

$$
\begin{aligned}
h_{n}(t)= & \sum_{i=1}^{m} \sum_{j=1}^{n} \frac{\gamma_{i} b_{j}}{\left(1-\sum_{i=1}^{m} \gamma_{i} \eta_{i}^{\alpha-1}\right) \Gamma\left(\alpha-\beta_{j}\right)} \int_{0}^{\eta_{i}}\left(\eta_{i}-s\right)^{\alpha-\beta_{j}-1} t^{\alpha-1} h_{n}(s) \mathrm{d} s \\
& -\sum_{i=1}^{n} \frac{b_{i}}{\left(1-\sum_{i=1}^{m} \gamma_{i} \eta_{i}^{\alpha-1}\right) \Gamma\left(\alpha-\beta_{i}\right)} \int_{0}^{1}(1-s)^{\alpha-\beta_{i}-1} t^{\alpha-1} h_{n}(s) \mathrm{d} s \\
& +\sum_{i=1}^{n} \frac{b_{i}}{\Gamma\left(\alpha-\beta_{i}\right)} \int_{0}^{t}(t-s)^{\alpha-\beta_{i}-1} h_{n}(s) \mathrm{d} s+\frac{1}{\Gamma(\alpha)} \int_{0}^{t}(t-s)^{\alpha-1} f_{n}(s) \mathrm{d} s \\
& +\sum_{i=1}^{m} \frac{\gamma_{i}}{\left(1-\sum_{i=1}^{m} \gamma_{i} \eta_{i}^{\alpha-1}\right) \Gamma(\alpha)} \int_{0}^{\eta_{i}}\left(\eta_{i}-s\right)^{\alpha-1} t^{\alpha-1} f_{n}(s) \mathrm{d} s \\
& -\frac{1}{\left(1-\sum_{i=1}^{m} \gamma_{i} \eta_{i}^{\alpha-1}\right) \Gamma(\alpha)} \int_{0}^{1}(1-s)^{\alpha-1} t^{\alpha-1} f_{n}(s) \mathrm{d} s .
\end{aligned}
$$

We need to show that there exists $f_{*} \in S_{F, u}$ such that for each $t \in[0,1]$,

$$
\begin{aligned}
h_{*}(t)= & \sum_{i=1}^{m} \sum_{j=1}^{n} \frac{\gamma_{i} b_{j}}{\left(1-\sum_{i=1}^{m} \gamma_{i} \eta_{i}^{\alpha-1}\right) \Gamma\left(\alpha-\beta_{j}\right)} \int_{0}^{\eta_{i}}\left(\eta_{i}-s\right)^{\alpha-\beta_{j}-1} t^{\alpha-1} h_{*}(s) \mathrm{d} s \\
& -\sum_{i=1}^{n} \frac{b_{i}}{\left(1-\sum_{i=1}^{m} \gamma_{i} \eta_{i}^{\alpha-1}\right) \Gamma\left(\alpha-\beta_{i}\right)} \int_{0}^{1}(1-s)^{\alpha-\beta_{i}-1} t^{\alpha-1} h_{*}(s) \mathrm{d} s \\
& +\sum_{i=1}^{n} \frac{b_{i}}{\Gamma\left(\alpha-\beta_{i}\right)} \int_{0}^{t}(t-s)^{\alpha-\beta_{i}-1} h_{*}(s) \mathrm{d} s+\frac{1}{\Gamma(\alpha)} \int_{0}^{t}(t-s)^{\alpha-1} f_{*}(s) \mathrm{d} s \\
& +\sum_{i=1}^{m} \frac{\gamma_{i}}{\left(1-\sum_{i=1}^{m} \gamma_{i} \eta_{i}^{\alpha-1}\right) \Gamma(\alpha)} \int_{0}^{\eta_{i}}\left(\eta_{i}-s\right)^{\alpha-1} t^{\alpha-1} f_{*}(s) \mathrm{d} s \\
& -\frac{1}{\left(1-\sum_{i=1}^{m} \gamma_{i} \eta_{i}^{\alpha-1}\right) \Gamma(\alpha)} \int_{0}^{1}(1-s)^{\alpha-1} t^{\alpha-1} f_{*}(s) \mathrm{d} s .
\end{aligned}
$$

In (2.18), taking

$$
\begin{aligned}
g(t)= & \frac{1}{\Gamma(\alpha)} \int_{0}^{t}(t-s)^{\alpha-1} f(s) \mathrm{d} s+\sum_{i=1}^{m} \frac{\gamma_{i}}{\left(1-\sum_{i=1}^{m} \gamma_{i} \eta_{i}^{\alpha-1}\right) \Gamma(\alpha)} \int_{0}^{\eta_{i}}\left(\eta_{i}-s\right)^{\alpha-1} t^{\alpha-1} f(s) \mathrm{d} s \\
& -\frac{1}{\left(1-\sum_{i=1}^{m} \gamma_{i} \eta_{i}^{\alpha-1}\right) \Gamma(\alpha)} \int_{0}^{1}(1-s)^{\alpha-1} t^{\alpha-1} f(s) \mathrm{d} s .
\end{aligned}
$$


In view of Lemma 2.10, we know that for any $f \in L([0,1], \mathbb{R})$, the integral equation (2.18) has a unique solution, which we denote by $h_{f}$. Because of this, we can define the operator

$$
\begin{aligned}
\Phi: L([0,1], \mathbb{R}) & \longrightarrow C([0,1], \mathbb{R}), \\
f & \longmapsto h_{f} .
\end{aligned}
$$

It is easy to verify that the operator $\Phi$ is linear. On the other hand, we can get

$$
\begin{aligned}
\left\|h_{f}\right\| \leq & \frac{1}{(1-\lambda) \Gamma(\alpha)} \int_{0}^{1}(|t-s|)^{\alpha-1}|f(s)| \mathrm{d} s \\
& +\frac{1}{(1-\lambda)\left(1-\sum_{i=1}^{m} \gamma_{i} \eta_{i}^{\alpha-1}\right) \Gamma(\alpha)} \int_{0}^{1}(1-s)^{\alpha-1}|f(s)| \mathrm{d} s \\
& +\sum_{i=1}^{m} \frac{\gamma_{i}}{(1-\lambda)\left(1-\sum_{i=1}^{m} \gamma_{i} \eta_{i}^{\alpha-1}\right) \Gamma(\alpha)} \int_{0}^{\eta_{i}}\left(\eta_{i}-s\right)^{\alpha-1}|f(s)| \mathrm{d} s \\
\leq & \mu \int_{0}^{1}|f(s)| \mathrm{d} s,
\end{aligned}
$$

where

$$
\mu=\frac{1}{(1-\lambda) \Gamma(\alpha)}+\sum_{i=1}^{m} \frac{\gamma_{i} \eta_{i}^{\alpha-1}}{(1-\lambda)\left(1-\sum_{i=1}^{m} \gamma_{i} \eta_{i}^{\alpha-1}\right) \Gamma(\alpha)}+\frac{1}{(1-\lambda)\left(1-\sum_{i=1}^{m} \gamma_{i} \eta_{i}^{\alpha-1}\right) \Gamma(\alpha)}
$$

that is, $\|\Phi(f)\| \leq \mu\|f\|_{L^{1}}$, which implies $\Phi$ is continuous. From Lemma 2.7, it follows that $\Phi \circ S_{F, u}$ is a closed graph operator. Moreover, we know $h_{n} \in \Phi\left(S_{F, u_{n}}\right)$. Since $u_{n} \rightarrow u_{*}$, it follows from Lemma 2.7 that (3.11) holds for some $f_{*} \in S_{F, u_{*}}$.

Therefore, $N$ is a compact multivalued map, u.s.c. with convex closed values. As a consequence of Lemma 2.8, we immediately conclude that $N$ has a fixed-point $u$ which is a solution of the problem (1.5) and (1.6). The proof is complete.

As a direct corollary of Theorem 3.1, we can immediately obtain the following result when the nonlinearity $F$ has sublinear growth in the state variable.

Corollary 3.2. Suppose that (H1) and (H2) are satisfied. Then the problem (1.5) and (1.6) has at least one solution in $C([0,1], \mathbb{R})$, provided that

(H4) there exist functions $p(t), q(t), r(t), \theta(t) \in L([0,1], \mathbb{R})$, and $\sigma_{1}, \sigma_{2}, \sigma_{3} \in[0,1)$ such that

$$
\|F(t, u, v, w)\|_{p} \leq p(t)|u|^{\sigma_{1}}+q(t)|v|^{\sigma_{2}}+r(t)|u|^{\sigma_{3}}+\theta(t)
$$

for each $(t, u, v, w) \in[0,1] \times \mathbb{R} \times \mathbb{R} \times \mathbb{R}$. 
Proof. We only need to verify that (H4) implies (H3) in Theorem 3.1. Taking $\varphi(t)=p(t)|u|^{\sigma_{1}}+$ $q(t)|v|^{\sigma_{2}}+r(t)|u|^{\sigma_{3}}+\theta(t)$, it is easy to prove

$$
\begin{gathered}
\tau=\lim _{r \rightarrow \infty} \inf \int_{0}^{1} \frac{\max \left\{\varphi_{r}(s), \varphi_{\tilde{k} r}(s), \varphi_{\tilde{l} r}(s)\right\}}{r} \mathrm{~d} s=0, \\
\mu_{i}=\lim _{r \rightarrow \infty} \inf \int_{0}^{\eta_{i}} \frac{\left(\eta_{i}-s\right)^{\alpha-1} \max \left\{\varphi_{r}(s), \varphi_{\tilde{k} r}(s), \varphi_{\tilde{l} r}(s)\right\}}{r} \mathrm{~d} s=0, \\
\omega=\lim _{r \rightarrow \infty} \inf \int_{0}^{1} \frac{(1-s)^{\alpha-1} \max \left\{\varphi_{r}(s), \varphi_{\tilde{k} r}(s), \varphi_{\tilde{l} r}(s)\right\}}{r} \mathrm{~d} s=0,
\end{gathered}
$$

because of $\sigma_{1}, \sigma_{2}, \sigma_{3} \in[0,1)$. Then (H3) is satisfied. The proof is finished.

In the next part, we are concerned with the BVP (1.5) and (1.6) with nonconvex valued right-hand side. By using Covitz and Nadler fixed-point theorem, we obtain the following result.

Theorem 3.3. Assume that the following hypotheses hold:

(A1) $F:[0,1] \times \mathbb{R} \times \mathbb{R} \times \mathbb{R} \rightarrow P_{c l}(\mathbb{R}) ; t \rightarrow F(t, u, v, w)$ is measurable for each $u, v, w \in \mathbb{R}$;

(A2) There exist three functions $p, q, r \in L^{1}([0,1], \mathbb{R})$ such that for a.e. $t \in[0,1]$ and $u_{1}, u_{2}, v_{1}, v_{2}, w_{1}, w_{2} \in \mathbb{R}$,

$$
\begin{gathered}
H_{d}\left(F\left(t, u_{1}, v_{1}, w_{1}\right), F\left(t, u_{2}, v_{2}, w_{2}\right)\right) \leq p(t)\left|u_{1}-u_{2}\right|+q(t)\left|v_{1}-v_{2}\right|+r(t)\left|w_{1}-w_{2}\right| \\
d(0, F(t, 0,0,0)) \leq m(t) .
\end{gathered}
$$

Then the BVP (1.5) and (1.6) has at least one solution in $C([0,1], \mathbb{R})$ provided that

$$
\tilde{a}_{0}+\tilde{b}_{0}+\tilde{c}_{0}+\sum_{i=1}^{m+1} \frac{\tilde{a}_{i}+\tilde{k} \tilde{b}_{i}+\tilde{l}_{\tilde{c}_{i}}}{\left(1-\sum_{i=1}^{m} \gamma_{i} \eta_{i}^{\alpha-1}\right)}<1-\lambda
$$

where

$$
\begin{array}{ll}
\tilde{a}_{0}=\frac{1}{\Gamma(\alpha)} \int_{0}^{1} p(s) \mathrm{d} s, & \tilde{a}_{i}=\frac{1}{\Gamma(\alpha)} \int_{0}^{\eta_{i}}\left(\eta_{i}-s\right)^{\alpha-1} p(s) \mathrm{d} s, \quad i=1,2, \ldots, m+1, \\
\tilde{b}_{0}=\frac{1}{\Gamma(\alpha)} \int_{0}^{1} q(s) \mathrm{d} s, & \tilde{b}_{i}=\frac{1}{\Gamma(\alpha)} \int_{0}^{\eta_{i}}\left(\eta_{i}-s\right)^{\alpha-1} q(s) \mathrm{d} s, \quad i=1,2, \ldots, m+1, \\
\tilde{c}_{0}=\frac{1}{\Gamma(\alpha)} \int_{0}^{1} p(s) \mathrm{d} s, & \tilde{c}_{i}=\frac{1}{\Gamma(\alpha)} \int_{0}^{\eta_{i}}\left(\eta_{i}-s\right)^{\alpha-1} r(s) \mathrm{d} s, \quad i=1,2, \ldots, m+1,
\end{array}
$$

$\eta_{m+1}=1$ and $\lambda$ defined as in Lemma 2.10, $\tilde{k}, \tilde{l}$ defined by (3.1) in Theorem 3.1. 
Proof. Transform the problem into a fixed-point problem. Let $N: C([0,1], \mathbb{R}) \rightarrow$ $P(C([0,1], \mathbb{R})$ be defined as in Theorem 3.1. We shall show that $N$ satisfies the assumptions of Lemma 2.9. The proof will be given in two steps.

Step 1. $N(u) \in P_{c l}(C([0,1], \mathbb{R}))$ for each $u \in C([0,1], \mathbb{R})$.

Indeed, let $\left(u_{n}\right)_{n \geq 0} \in N(u)$ such that $u_{n} \rightarrow \tilde{u}$ in $C([0,1], \mathbb{R})$. Then there exists $g_{n} \in S_{F, u}$ such that for each $t \in[0,1]$,

$$
\begin{aligned}
u_{n}(t)= & \sum_{i=1}^{m} \sum_{j=1}^{n} \frac{\gamma_{i} b_{j}}{\left(1-\sum_{i=1}^{m} \gamma_{i} \eta_{i}^{\alpha-1}\right) \Gamma\left(\alpha-\beta_{j}\right)} \int_{0}^{\eta_{i}}\left(\eta_{i}-s\right)^{\alpha-\beta_{j}-1} t^{\alpha-1} u_{n}(s) \mathrm{d} s \\
& -\sum_{i=1}^{n} \frac{b_{i}}{\left(1-\sum_{i=1}^{m} \gamma_{i} \eta_{i}^{\alpha-1}\right) \Gamma\left(\alpha-\beta_{i}\right)} \int_{0}^{1}(1-s)^{\alpha-\beta_{i}-1} t^{\alpha-1} u_{n}(s) \mathrm{d} s \\
& +\sum_{i=1}^{n} \frac{b_{i}}{\Gamma\left(\alpha-\beta_{i}\right)} \int_{0}^{t}(t-s)^{\alpha-\beta_{i}-1} u_{n}(s) \mathrm{d} s+\frac{1}{\Gamma(\alpha)} \int_{0}^{t}(t-s)^{\alpha-1} g_{n}(s) \mathrm{d} s \\
& +\sum_{i=1}^{m} \frac{\gamma_{i}}{\left(1-\sum_{i=1}^{m} \gamma_{i} \eta_{i}^{\alpha-1}\right) \Gamma(\alpha)} \int_{0}^{\eta_{i}}\left(\eta_{i}-s\right)^{\alpha-1} t^{\alpha-1} g_{n}(s) \mathrm{d} s \\
& -\frac{1}{\left(1-\sum_{i=1}^{m} \gamma_{i} \eta_{i}^{\alpha-1}\right) \Gamma(\alpha)} \int_{0}^{1}(1-s)^{\alpha-1} t^{\alpha-1} g_{n}(s) \mathrm{d} s .
\end{aligned}
$$

For every $w \in F(t, u(t),(T u)(t),(S u)(t))$, from (A2), we have

$$
|w| \leq d(0, F(t, 0,0,0))+H_{d}(F(t, 0,0,0), F(t, u(t),(T u)(t),(S u)(t))) .
$$

Then,

$$
\left|g_{n}(t)\right| \leq m(t)+p(t)|u(t)|+q(t)|(T u)(t)|+r(t)|(S u)(t)| \quad \text { a.e. } t \in[0,1],
$$

that is, $g_{n}(t) \in B(0, m(t)+p(t)|u(t)|+q(t)|(T u)(t)|+r(t)|(S u)(t)|)$, where

$$
\begin{aligned}
B(0, & m(t)+p(t)|u(t)|+q(t)|(T u)(t)|+r(t)|(S u)(t)|) \\
\quad & =\{w \in \mathbb{R}:|w| \leq m(t)+p(t)|u(t)|+q(t)|(T u)(t)|+r(t)|(S u)(t)|\}:=\Psi(t) .
\end{aligned}
$$

It is clear that $\Psi:[0,1] \rightarrow p_{c p, c v}(\mathbb{R})$ is a multivalued integrable bounded map. Since $g_{n}(\cdot) \in \Psi(\cdot), n \geq 1$, we may pass to a subsequence if necessary to get that $g_{n}$ converges weakly to $g$ in $L_{w}^{1}([0,1], \mathbb{R})$. From Mauz's lemma [37], there exists $g \in \overline{\operatorname{conv}}\left\{g_{n}(t): n \geq 1\right\}$, then there 
exists a subsequence $\left\{\bar{g}_{n}: n \geq 1\right\}$ in $\overline{\operatorname{conv}}\left\{g_{n}: n \geq 1\right\}$, such that $\bar{g}_{n}$ converges strongly to $g$ in $L^{1}([0,1], \mathbb{R})$, which implies $g \in L^{1}([0,1], \mathbb{R})$. Then for each $t \in[0,1]$,

$$
\begin{aligned}
u_{n}(t) \longrightarrow \tilde{u}(t)= & \sum_{i=1}^{m} \sum_{j=1}^{n} \frac{\gamma_{i} b_{j}}{\left(1-\sum_{i=1}^{m} \gamma_{i} \eta_{i}^{\alpha-1}\right) \Gamma\left(\alpha-\beta_{j}\right)} \int_{0}^{\eta_{i}}\left(\eta_{i}-s\right)^{\alpha-\beta_{j}-1} t^{\alpha-1} \tilde{u}(s) \mathrm{d} s \\
& -\sum_{i=1}^{n} \frac{b_{i}}{\left(1-\sum_{i=1}^{m} \gamma_{i} \eta_{i}^{\alpha-1}\right) \Gamma\left(\alpha-\beta_{i}\right)} \int_{0}^{1}(1-s)^{\alpha-\beta_{i}-1} t^{\alpha-1} \tilde{u}(s) \mathrm{d} s \\
& +\sum_{i=1}^{n} \frac{b_{i}}{\Gamma\left(\alpha-\beta_{i}\right)} \int_{0}^{t}(t-s)^{\alpha-\beta_{i}-1} \tilde{u}(s) \mathrm{d} s+\frac{1}{\Gamma(\alpha)} \int_{0}^{t}(t-s)^{\alpha-1} g(s) \mathrm{d} s \\
& +\sum_{i=1}^{m} \frac{\gamma_{i}}{\left(1-\sum_{i=1}^{m} \gamma_{i} \eta_{i}^{\alpha-1}\right) \Gamma(\alpha)} \int_{0}^{\eta_{i}}\left(\eta_{i}-s\right)^{\alpha-1} t^{\alpha-1} g(s) \mathrm{d} s \\
& -\frac{1}{\left(1-\sum_{i=1}^{m} \gamma_{i} \eta_{i}^{\alpha-1}\right) \Gamma(\alpha)} \int_{0}^{1}(1-s)^{\alpha-1} t^{\alpha-1} g(s) \mathrm{d} s .
\end{aligned}
$$

So, $\tilde{u} \in N(u) \in p_{c l}(C([0,1], \mathbb{R}))$.

Step 2. There exists $\gamma<1$, such that

$$
H_{d}\left(N(u), N\left(u_{*}\right)\right) \leq \gamma\left\|u-u_{*}\right\|_{\infty} \text { for each } u, u_{*} \in C([0,1], \mathbb{R}) .
$$

Let $u, u_{*} \in C([0,1], \mathbb{R})$ and $h \in N(u)$. Then there exists $f(t) \in F(t, u(t),(T u)(t),(S u)(t))$ such that for each $t \in[0,1]$

$$
\begin{aligned}
h(t)= & \sum_{i=1}^{m} \sum_{j=1}^{n} \frac{\gamma_{i} b_{j}}{\left(1-\sum_{i=1}^{m} \gamma_{i} \eta_{i}^{\alpha-1}\right) \Gamma\left(\alpha-\beta_{j}\right)} \int_{0}^{\eta_{i}}\left(\eta_{i}-s\right)^{\alpha-\beta_{j}-1} t^{\alpha-1} h(s) \mathrm{d} s \\
& -\sum_{i=1}^{n} \frac{b_{i}}{\left(1-\sum_{i=1}^{m} \gamma_{i} \eta_{i}^{\alpha-1}\right) \Gamma\left(\alpha-\beta_{i}\right)} \int_{0}^{1}(1-s)^{\alpha-\beta_{i}-1} t^{\alpha-1} h(s) \mathrm{d} s \\
& +\sum_{i=1}^{n} \frac{b_{i}}{\Gamma\left(\alpha-\beta_{i}\right)} \int_{0}^{t}(t-s)^{\alpha-\beta_{i}-1} h(s) \mathrm{d} s+\frac{1}{\Gamma(\alpha)} \int_{0}^{t}(t-s)^{\alpha-1} f(s) \mathrm{d} s \\
& +\sum_{i=1}^{m} \frac{\gamma_{i}}{\left(1-\sum_{i=1}^{m} \gamma_{i} \eta_{i}^{\alpha-1}\right) \Gamma(\alpha)} \int_{0}^{\eta_{i}}\left(\eta_{i}-s\right)^{\alpha-1} t^{\alpha-1} f(s) \mathrm{d} s \\
& -\frac{1}{\left(1-\sum_{i=1}^{m} \gamma_{i} \eta_{i}^{\alpha-1}\right) \Gamma(\alpha)} \int_{0}^{1}(1-s)^{\alpha-1} t^{\alpha-1} f(s) \mathrm{d} s .
\end{aligned}
$$


It follows from (A2) that

$$
\begin{aligned}
& H_{d}\left(F(t, u(t),(T u)(t),(S u)(t)), F\left(t, u_{*}(t),\left(T u_{*}\right)(t),\left(S u_{*}\right)(t)\right)\right) \\
& \quad \leq p(t)\left|u(t)-u_{*}(t)\right|+q(t)\left|(T u)(t)-\left(T u_{*}\right)(t)\right|+r(t)\left|(S u)(t)-\left(S u_{*}\right)(t)\right|, \quad t \in[0,1] .
\end{aligned}
$$

Hence, there is $g \in F\left(t, u_{*}(t),\left(T u_{*}\right)(t),\left(S u_{*}\right)(t)\right)$ such that

$$
|f(t)-g| \leq p(t)\left|u(t)-u_{*}(t)\right|+q(t)\left|(T u)(t)-\left(T u_{*}\right)(t)\right|+r(t)\left|(S u)(t)-\left(S u_{*}\right)(t)\right|, \quad t \in[0,1] .
$$

Consider $G:[0,1] \rightarrow D(\mathbb{R})$, given by

$G(t)$

$$
=\left\{g \in \mathbb{R}:|f(t)-g| \leq p(\mathrm{t})\left|u(t)-u_{*}(t)\right|+q(t)\left|(T u)(t)-\left(T u_{*}\right)(t)\right|+r(t)\left|(S u)(t)-\left(S u_{*}\right)(t)\right|\right\} .
$$

Since the multivalued operator $\tilde{G}: t \rightarrow G(t) \cap F\left(t, u_{*}(t),\left(T u_{*}\right)(t),\left(S u_{*}\right)(t)\right)$ is measurable (see in [38, Proposition III.4]), there exists a function $g_{*}$, which is a measurable selection for $G$. So, $g_{*}(t) \in F\left(t, u_{*}(t),\left(T u_{*}\right)(t),\left(S u_{*}\right)(t)\right)$ and

$$
\begin{aligned}
\mid f(t) & -g_{*}(t) \mid \\
& \leq p(t)\left|u(t)-u_{*}(t)\right|+q(t)\left|(T u)(t)-\left(T u_{*}\right)(t)\right|+r(t)\left|(S u)(t)-\left(S u_{*}\right)(t)\right|, \quad t \in[0,1] .
\end{aligned}
$$

For each $t \in[0,1]$, define

$$
\begin{aligned}
h_{*}(t)= & \sum_{i=1}^{m} \sum_{j=1}^{n} \frac{\gamma_{i} b_{j}}{\left(1-\sum_{i=1}^{m} \gamma_{i} \eta_{i}^{\alpha-1}\right) \Gamma\left(\alpha-\beta_{j}\right)} \int_{0}^{\eta_{i}}\left(\eta_{i}-s\right)^{\alpha-\beta_{j}-1} t^{\alpha-1} h_{*}(s) \mathrm{d} s \\
& -\sum_{i=1}^{n} \frac{b_{i}}{\left(1-\sum_{i=1}^{m} \gamma_{i} \eta_{i}^{\alpha-1}\right) \Gamma\left(\alpha-\beta_{i}\right)} \int_{0}^{1}(1-s)^{\alpha-\beta_{i}-1} t^{\alpha-1} h_{*}(s) \mathrm{d} s \\
& +\sum_{i=1}^{n} \frac{b_{i}}{\Gamma\left(\alpha-\beta_{i}\right)} \int_{0}^{t}(t-s)^{\alpha-\beta_{i}-1} h_{*}(s) \mathrm{d} s+\frac{1}{\Gamma(\alpha)} \int_{0}^{t}(t-s)^{\alpha-1} g_{*}(s) \mathrm{d} s \\
& +\sum_{i=1}^{m} \frac{\gamma_{i}}{\left(1-\sum_{i=1}^{m} \gamma_{i} \eta_{i}^{\alpha-1}\right) \Gamma(\alpha)} \int_{0}^{\eta_{i}}\left(\eta_{i}-s\right)^{\alpha-1} t^{\alpha-1} g_{*}(s) \mathrm{d} s \\
& -\frac{1}{\left(1-\sum_{i=1}^{m} \gamma_{i} \eta_{i}^{\alpha-1}\right) \Gamma(\alpha)} \int_{0}^{1}(1-s)^{\alpha-1} t^{\alpha-1} g_{*}(s) \mathrm{d} s .
\end{aligned}
$$


Then, for $t \in[0,1]$

$$
\begin{aligned}
\left|h(t)-h_{*}(t)\right| \leq & \sum_{i=1}^{m} \sum_{j=1}^{n} \frac{\gamma_{i} b_{j}}{\left(1-\sum_{i=1}^{m} \gamma_{i} \eta_{i}^{\alpha-1}\right) \Gamma\left(\alpha-\beta_{j}\right)} \int_{0}^{\eta_{i}}\left(\eta_{i}-s\right)^{\alpha-\beta_{j}-1} t^{\alpha-1}\left|h(s)-h_{*}(s)\right| \mathrm{d} s \\
& +\sum_{i=1}^{n} \frac{b_{i}}{\left(1-\sum_{i=1}^{m} \gamma_{i} \eta_{i}^{\alpha-1}\right) \Gamma\left(\alpha-\beta_{i}\right)} \int_{0}^{1}(1-s)^{\alpha-\beta_{i}-1} t^{\alpha-1}\left|h(s)-h_{*}(s)\right| \mathrm{d} s \\
& +\sum_{i=1}^{n} \frac{b_{i}}{\Gamma\left(\alpha-\beta_{i}\right)} \int_{0}^{t}(t-s)^{\alpha-\beta_{i}-1}\left|h(s)-h_{*}(s)\right| \mathrm{d} s \\
& +\sum_{i=1}^{m} \frac{\gamma_{i}}{\left(1-\sum_{i=1}^{m} \gamma_{i} \eta_{i}^{\alpha-1}\right) \Gamma(\alpha)} \int_{0}^{\eta_{i}}\left(\eta_{i}-s\right)^{\alpha-1} t^{\alpha-1}\left|f(s)-g_{*}(s)\right| \mathrm{d} s \\
& +\frac{1}{\left(1-\sum_{i=1}^{m} \gamma_{i} \eta_{i}^{\alpha-1}\right) \Gamma(\alpha)} \int_{0}^{1}(1-s)^{\alpha-1} t^{\alpha-1}\left|f(s)-g_{*}(s)\right| \mathrm{d} s \\
& +\frac{1}{\Gamma(\alpha)} \int_{0}^{t}(t-s)^{\alpha-1}\left|f(s)-g_{*}(s)\right| \mathrm{d} s \\
\leq & \lambda\left\|h-h_{*}\right\|+\left\|u-u_{*}\right\| \\
& \times\left(\frac{1}{\Gamma(\alpha)} \int_{0}^{t}(t-s)^{\alpha-1}(p(s)+q(s) \tilde{k}+r(s) \tilde{l}) \mathrm{d} s\right. \\
& +\sum_{i=1}^{m} \frac{\gamma_{i}}{\left(1-\sum_{i=1}^{m} \gamma_{i} \eta_{i}^{\alpha-1}\right) \Gamma(\alpha)} \int_{0}^{\eta_{i}}\left(\eta_{i}-s\right)^{\alpha-1} t^{\alpha-1}(p(s)+q(s) \tilde{k}+r(s) \tilde{l}) \mathrm{d} s \\
& \left.+\frac{1}{\left(1-\sum_{i=1}^{m} \gamma_{i} \eta_{i}^{\alpha-1}\right) \Gamma(\alpha)} \int_{0}^{1}(1-s)^{\alpha-1} t^{\alpha-1}(p(s)+q(s) \tilde{k}+r(s) \tilde{l}) \mathrm{d} s\right) .
\end{aligned}
$$

So,

$$
\left\|h-h_{*}\right\| \leq\left(\frac{\tilde{a}_{0}+\tilde{b}_{0}+\tilde{c}_{0}}{1-\lambda}+\sum_{i=1}^{m+1} \frac{\tilde{a}_{i}+\tilde{k} \tilde{b}_{i}+\tilde{l}_{\tilde{c}_{i}}}{(1-\lambda)\left(1-\sum_{i=1}^{m} \gamma_{i} \eta_{i}^{\alpha-1}\right)}\right)\left\|u-u_{*}\right\| .
$$

By an analogous relation, obtained by interchanging the roles of $u, u_{*}$, it follows that

$$
H_{d}\left(N(u), N\left(u_{*}\right)\right) \leq C\left\|u-u_{*}\right\|
$$

where $C=\left(\tilde{a}_{0}+\tilde{b}_{0}+\widetilde{c}_{0}\right) /(1-\lambda)+\sum_{i=1}^{m+1}\left(\tilde{a}_{i}+\tilde{k} \tilde{b}_{i}+\tilde{c}_{i} /(1-\lambda)\left(1-\sum_{i=1}^{m} \gamma_{i} \eta_{i}^{\alpha-1}\right)\right)<1$. Therefore, $N$ is a contraction operator. With the help of Lemma 2.9, the problem (1.5) and (1.6) has at least one solution. 
Remark 3.4. In (1.5) and (1.6), if $b_{i}=0, \gamma_{j}=0, i=1, \ldots, n, j=1, \ldots, m, k(t, s)=l(t, s) \equiv 0$. Then we obtain the following fractional boundary value problem of differential inclusions

$$
\begin{gathered}
-D^{\alpha} y(t) \in F(t, y(t)), \quad \text { a.e. } t \in J=[0,1], 0<\alpha \leq 2, \\
y(0)=y(1)=0
\end{gathered}
$$

which has been studied in [26]. It is easy to see that our results (Theorems 3.1 and 3.3) partly generalize and improve the results obtained in it.

\section{Acknowledgments}

This work is supported by the National Natural Science Foundation of PR China (no. 10701023, No. 10971221) and Shanghai Natural Science Foundation (no. 10ZR1400100).

\section{References}

[1] R. W. Ibrahim and S. Momani, "On the existence and uniqueness of solutions of a class of fractional differential equations," Journal of Mathematical Analysis and Applications, vol. 334, no. 1, pp. 1-10, 2007.

[2] A. Babakhani and V. Daftardar-Gejji, "Existence of positive solutions of nonlinear fractional differential equations," Journal of Mathematical Analysis and Applications, vol. 278, no. 2, pp. 434-442, 2003.

[3] K. Deimling, Multivalued Differential Equations, Walter de Gruyter, Berlin, Germany, 1992.

[4] V. Lakshmikantham, S. Leela, and J. Vasundhara, Theory of Fractional Dynamic Systems, Cambridge Academic, Cambridge, UK, 2009.

[5] V. Lakshmikantham and A. S. Vatsala, "Basic theory of fractional differential equations," Nonlinear Analysis. Theory, Methods \& Applications, vol. 69, no. 8, pp. 2677-2682, 2008.

[6] C. Yu and G. Gao, "Existence of fractional differential equations," Journal of Mathematical Analysis and Applications, vol. 310, no. 1, pp. 26-29, 2005.

[7] A. Babakhani and V. D. Gejji, "Existence of positive solutions of nonlinear fractional differential equations," Journal of Mathematical Analysis and Applications, vol. 278, no. 2, pp. 434-442, 2003.

[8] K. S. Miller and B. Ross, An Introduction to the Fractional Calculus and Fractional Differential Equations, John Wiley \& Sons, New York, NY, USA, 1993.

[9] I. Podlubny, Fractional Differential Equations: An Introduction to Fractional Derivatives, Fractional Differential Equations, to Methods of Their Solution and Some of Their Application, vol. 198, Academic Press, San Diego, Calif, USA, 1999.

[10] D. Delbosco and L. Rodino, "Existence and uniqueness for a nonlinear fractional differential equation," Journal of Mathematical Analysis and Applications, vol. 204, no. 2, pp. 609-625, 1996.

[11] M. Stojanović, "Existence-uniqueness result for a nonlinear -term fractional equation," Journal of Mathematical Analysis and Applications, vol. 353, no. 1, pp. 244-255, 2009.

[12] A. Arara, M. Benchohra, N. Hamidi, and J. J. Nieto, "Fractional order differential equations on an unbounded domain," Nonlinear Analysis. Theory, Methods E Applications, vol. 72, no. 2, pp. 580-586, 2010.

[13] A. M. A. El-Sayed and A. G. Ibrahim, "Multivalued fractional differential equations," Applied Mathematics and Computation, vol. 68, no. 1, pp. 15-25, 1995.

[14] R. P. Agarwal, M. Belmekki, and M. Benchohra, "A survey on semilinear differential equations and inclusions involving Riemann-Liouville fractional derivative," Advances in Difference Equations, vol. 2009, Article ID 981728, 47 pages, 2009.

[15] R. P. Agarwal, M. Belmekki, and M. Benchohra, "Existence results for semilinear functional differential inclusions involving Riemann-Liouville fractional derivative," Dynamics of Continuous, Discrete $\mathcal{E}$ Impulsive Systems. Series A, vol. 17, no. 3, pp. 347-361, 2010. 
[16] R. P. Agarwal, M. Benchohra, and S. Hamani, "A survey on existence results for boundary value problems of nonlinear fractional differential equations and inclusions," Acta Applicandae Mathematicae, vol. 109, no. 3, pp. 973-1033, 2010.

[17] M. Benchohra, J. R. Graef, and S. Hamani, "Existence results for boundary value problems with nonlinear fractional differential equations," Applicable Analysis, vol. 87, no. 7, pp. 851-863, 2008.

[18] M. Benchohra, J. Henderson, S. K. Ntouyas, and A. Ouahab, "Existence results for fractional functional differential inclusions with infinite delay and applications to control theory," Fractional Calculus \& Applied Analysis, vol. 11, no. 1, pp. 35-56, 2008.

[19] M. Benchohra, J. R. Graef, and S. Hamani, "Existence results for boundary value problems with nonlinear fractional differential equations," Applicable Analysis, vol. 87, no. 7, pp. 851-863, 2008.

[20] R. P. Agarwal, M. Benchohra, and S. Hamani, "Boundary value problems for differential inclusions with fractional order," Advanced Studies in Contemporary Mathematics, vol. 16, no. 2, pp. 181-196, 2008.

[21] B. Ahmad, "Existence of solutions for fractional differential equations of order $q \in(2,3]$ with antiperiodic boundary conditions," Journal of Applied Mathematics and Computing, vol. 34, no. 1-2, pp. 385-391, 2010.

[22] J. Henderson and A. Ouahab, "Fractional functional differential inclusions with finite delay," Nonlinear Analysis. Theory, Methods E Applications, vol. 70, no. 5, pp. 2091-2105, 2009.

[23] A. Lin and L. Hu, "Existence results for impulsive neutral stochastic functional integro-differential inclusions with nonlocal initial conditions," Computers \& Mathematics with Applications, vol. 59, no. 1, pp. 64-73, 2010.

[24] J. Henderson and A. Ouahab, "Impulsive differential inclusions with fractional order," Computers $\mathcal{E}$ Mathematics with Applications, vol. 59, no. 3, pp. 1191-1226, 2010.

[25] N. Abada, M. Benchohra, and H. Hammouche, "Existence and controllability results for nondensely defined impulsive semilinear functional differential inclusions," Journal of Differential Equations, vol. 246, no. 10, pp. 3834-3863, 2009.

[26] A. Ouahab, "Some results for fractional boundary value problem of differential inclusions," Nonlinear Analysis. Theory, Methods \& Applications., vol. 69, no. 11, pp. 3877-3896, 2008.

[27] Y.-K. Chang and J. J. Nieto, "Some new existence results for fractional differential inclusions with boundary conditions," Mathematical and Computer Modelling, vol. 49, no. 3-4, pp. 605-609, 2009.

[28] G. Li and X. Xue, "On the existence of periodic solutions for differential inclusions," Journal of Mathematical Analysis and Applications, vol. 276, no. 1, pp. 168-183, 2002.

[29] R. M. Colombo, A. Fryszkowski, T. Rzeżuchowski, and V. Staicu, "Continuous selections of solution sets of Lipschitzean differential inclusions," Funkcialaj Ekvacioj. Serio Internacia, vol. 34, no. 2, pp. 321330, 1991.

[30] H. F. Bohnenblust and S. Karlin, "On a theorem of Ville," in Contributions to the Theory of Games, vol. 1, pp. 155-160, Princeton University, Princeton, NJ, USA, 1950.

[31] M. Kisielewicz, Differential Inclusions and Optimal Control, Kluwer Academic Publishers, Dordrecht, The Netherlands, 1991.

[32] L. Górniewicz, "Topological fixed point theory of multivalued mappings," in Mathematics and Its Applications, vol. 495, Kluwer Academic Publishers, Dordrecht, The Netherlands, 1999.

[33] S. Hu and N. S. Papageorgiou, Handbook of Multivalued Analysis. Vol. I, vol. 419 of Mathematics and Its Applications, Kluwer Academic Publishers, Dordrecht, The Netherlands, 1997.

[34] A. A. Tolstonogov, Differential Inclusions in a Banach Space, Kluwer Academic Publishers, Dordrecht, The Netherlands, 2000.

[35] A. Lasota and Z. Opial, "An application of the Kakutani-Ky Fan theorem in the theory of ordinary differential equations," Bulletin de l'Académie Polonaise des Sciences. Série des Sciences Mathématiques, Astronomiques et Physiques, vol. 13, pp. 781-786, 1965.

[36] H. Covitz and S. B. Nadler,, "Multi-valued contraction mappings in generalized metric spaces," Israel Journal of Mathematics, vol. 8, pp. 5-11, 1970.

[37] P. D. Lax, Functional Analysis, John Wiley \& Sons, New York, NY, USA, 2002.

[38] C. Castaing and M. Valadier, "Convex analysis and measurable multifunctions," in Lecture Notes in Mathematics, vol. 580, Springer, Berlin, Germany, 1977. 


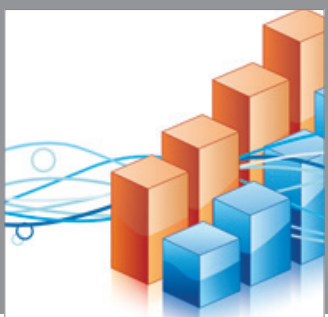

Advances in

Operations Research

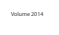

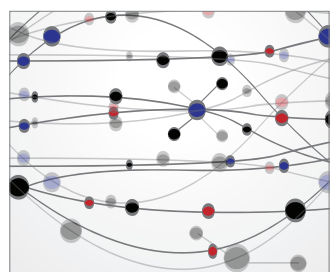

\section{The Scientific} World Journal
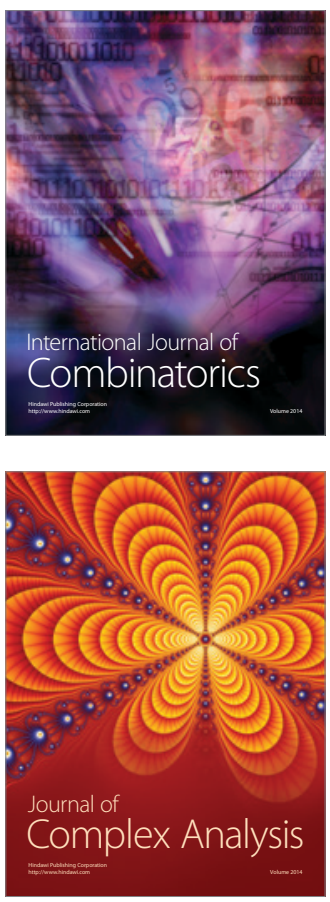

International Journal of

Mathematics and

Mathematical

Sciences
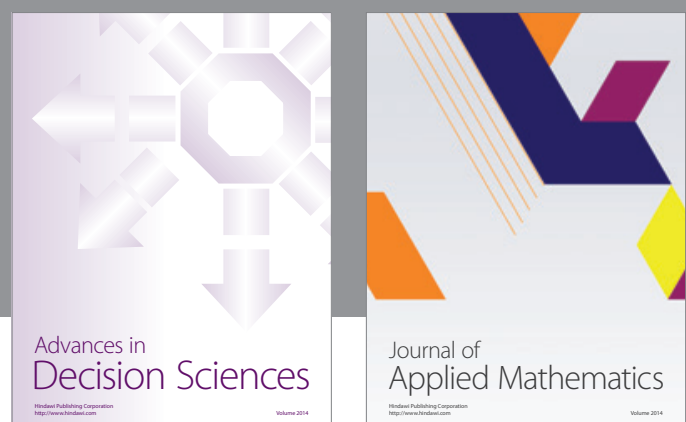

Journal of

Applied Mathematics
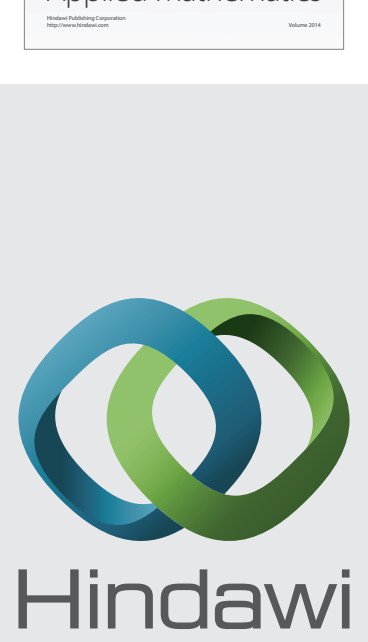

Submit your manuscripts at http://www.hindawi.com


Mathematical Problems in Engineering


Journal of

Function Spaces
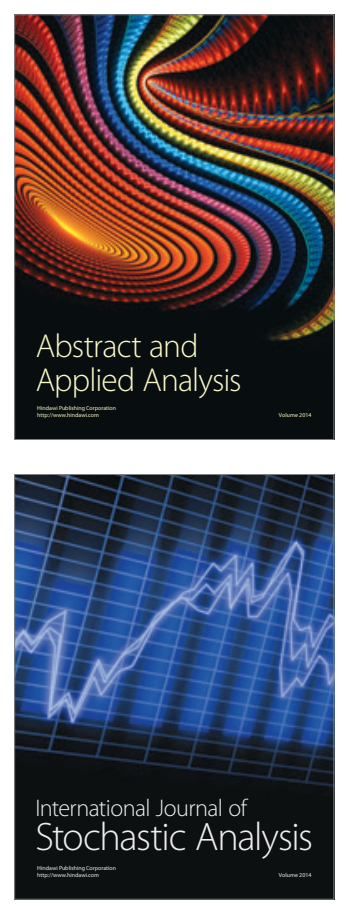

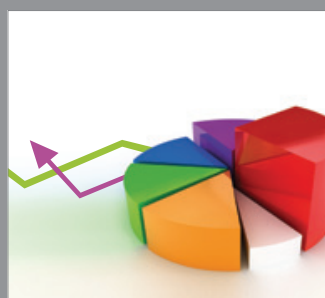

ournal of

Probability and Statistics

Promensencen
